\title{
Mechanical Designing of Acoustic Chamber Based Muffler for Sound Reduction
}

\author{
Sandeep Yadav, Rituraj Raghuvanshi
}

\begin{abstract}
Sound of exhaust system makes a substantial contribution to the interior as well as exterior noise of automobiles. Accurate prediction and control of noise from automobiles is of significant importance in automotive exhaust system design. When it comes into the term of acoustic performance there are several parameters that describe the performance of a muffler, the Transmission Loss (TL) being the most popular one for validating an analytical model. Acoustic characteristics of silencers filled with fibrous material are also studied.
\end{abstract}

Keywords: Transmission Loss (TL), Reactive Muffler, Central Inlet and Side Outlet, Multi Expansion Chamber, Dissipative Muffler, Hybrid Muffler, Sound Quality.

\section{INTRODUCTION}

The physics word "ACOUSTIC" is derived from the Greek word akoustikos meaning "of or for hearing, ready to hear" and that from akoustos, "heard, audible", which in turn derives from the verb akouo, "I hear", The Latin synonym is "sonic", after which the term sonics used to be a synonym for acoustics and later a branch of acoustics , Frequencies above and below the audible range are called "ultrasonic" and "infrasonic", respectively. Pythagoras explained the rationale behind as to why some intervals is perceived to be more delectable than others. The answer to this query was that when the ratio of the vibrating strings is in integers (say, 2 to 3 or 3 to 4), the tones produced will be harmonious. He further emphasized that the universe evolves from sound a statement which is very closely associated with the traditional Vedic belief of the ancient Indian civilization if available sound were replaced with cosmic vibrations. Aristotle (384 - 322 BC) seemed to have identified the mechanism of sound propagation as a series of expansions and contractions of air, an observation which is very true to the classical theory of acoustic wave propagation. Vitruvius, the Roman architect and engineer wrote a treatise on the acoustic properties of theatres including elaborate discussions on interference, echo and reverberation - the concepts taught in modern architectural acoustics.

\section{LITERATURE REVIEW}

In this work the maker recommend that a compelled portion move toward is appeared for showing sound development in punctured dissipative silencers with non-uniform property.

Revised Manuscript Received on February 05, 2020.

* Correspondence Author

Sandeep Yadav, Assistant Professor, Department of Fire Technology \& Safety Engineering Medi-Caps University, Indore, India.

Rituraj Raghuvanshi, Assistant Professor, Department of Fire Technology \& Safety Engineering Medi-Caps University, Indore, India

(c) The Authors. Published by Blue Eyes Intelligence Engineering and Sciences Publication (BEIESP). This is an open access article under the CC BY-NC-ND license (http://creativecommons.org/licenses/by-nc-nd/4.0/)
The spatial combinations of the properties can create, for instance, from uneven satisfying outlines in the midst of make and debasement related with the movement of development particles inside the retentive material. In any case, the constrained segment system is associated with the wave condition for a multiplication medium with variable properties (outside chamber with retentive material) and a uniform medium (central area). For the 31 occurrence of a dissipative silencers, the depiction of the elastic materials is finished by techniques for its equivalent complex thickness and speed of sound. A.G Antebas,F.D. Denia*, A.M. Pedrosa, F.J.Fuenmayor.The maker's essential objective for this work is to separate the introduction of by far the majority of the silencers with/without noise elastic material. Under the statement of flat wave inducing, the trade networks among the two terminations of plane pipe and 2pipe punctured zone are gathered and taken as the essential segments; base on the situation of cognizance of weight and of mass speed, the silencer is shown as a framework a confined by the two key segments. By then the noise decreasing typical for the silencers can be investigate. With this arrangement the copy related channels can in like manner be analyze. Further, the absorbent sound light substance is in like manner consolidated into this arrangement. The effect of sound maintenance material on the presentation of silencers is dismembered and discussed. Chao-Nan Wanga, * Chien-Ho Wua, Tzann-Dwo Wub

\section{RESEARCH ANALYSIS}

\section{Muffler Selection and Design Parameters}

Determine the fumes stream and worthy fumes framework backpressure of motor.

A free-streaming air admission and fumes framework in vehicle.

Muffler must be assembled hard to deal with high weight exhaust gasses, retain sway from street trash, and oppose erosion.

Number of deltas, single or double framework.

Diameter of pipe, Inlet and outlet.

Size of the suppressor.

Material used, tempered steel silencer offers unrivaled erosion opposition, quality, and future than the aluminized steel silencer.

Number of chambers

Number of channels and outlet pipes

Diameter of Inlet and outlet pipe

Holes on the pipe Size of silencer 


\section{Mechanical Designing of Acoustic Chamber Based Muffler for Sound Reduction}

\section{Muffler Performance Parameters}

\section{Insertion Loss}

Inclusion misfortune is a term used to depict the qualification in sound weight level while melding a change to a given structure; it is used to check the impact of sound reducing measures. In the image underneath the sound weight level is changed when the pipe is stretched out with its impact being the inclusion

misfortune. On the off chance that W1 and W2 are control transmitted without and with channel individually, at that point,

$\mathrm{IL}=\mathrm{LW} 1-\mathrm{LW} 2=10 \log (\mathrm{W} 1 / \mathrm{W} 2) \mathrm{dB}$

\section{Transmission Loss}

Transmission misfortune is the association between the information influence and the transmitted influence from a shield or segment in a pipe structure. It is portrayed in the condition underneath. The transmission can't be direct evaluated since sound power itself can't be assessed.

$\mathrm{TL}=10 \log (\mathrm{An} / \mathrm{A} 1) \mathrm{B} 1=0$

$\mathrm{An}=$ Amplitude of episode weight waves in the exhaust pipe A1 = Amplitude of episode weight waves inside the tail pipe B1 =Amplitude of reflected weight waves inside the tail pipe Transmission misfortunes and inclusion misfortunes are both used while delineating sound decreasing measures anyway the transmission misfortune is increasingly common and is associated more to the sound influence which is generally better to use. Transmission misfortune from a couple of sources can some of the time be superposed together with a particular ultimate objective to get the total transmission misfortune which is regularly what one is looking for.

\section{Flow Resistance and Flow Resistivity}

Stream resistivity is a proportion of the material's obstruction from being infiltrated by a sound wave (Ray, 2010). Low estimations of stream resistivity infer that a sound wave will successfully infiltrate a material which thus will extend debilitating through the material. Low estimations of stream resistivity are helpful for debilitating high frequencies while low 76 characteristics are valuable for contracting lower frequencies, suggesting that there are trade offs while picking confound material. Stream resistivity is delicate to changes in gas consistency, taking off it easily affected by enhancements to working temperatures. In spite of the way that it's definitely not a real acoustic sum it is used comprehensively in the source material for this report as a sum that chooses the properties of the damping material in the silencers. Stream opposition isn't an undefined parameter from stream resistivity. Resistivity is associated with the properties of the material while stream opposition is a specific parameter for a particular plan of bewilders with a specific stream resistivity.

\section{DIL - Dynamic Insertion Loss}

The Dynamic Insertion Loss-DIL is the complexity between the sound power or force levels evaluated in a comparable motivation behind the ventilation work when the consideration of the silencer. The addition misfortunes depend upon the stream - if it's forward or pivot. The stream is forward if wind streams a similar way as the spread of sound.

\section{SN - Self Noise}

The Self Noise-SN is the Noise control level in decibels created by the silencer when installed in the wind stream. The Self Noise depends upon the course of the stream - if it's forward or invert.

\section{Materials utilized for Silencer}

Fibrous Materials \& Stringy materials will be materials which fundamentally utilized for applications where there is must need to ingest sound. Stringy materials have great sound engrossing coefficient that they are valuable in silencers and in structure developments where there is must need to decrease sound dimension. There is different sound engrossing materials essentially utilized which has high solid retaining coefficient.

Different Types of Fibrous Materials:- Acoustic Belts \& tiles, Asbestos, Sprayed, Brickwork painted Brickwork unpainted, Cork sheet, Fiber board on secures (Synthetic Fiber),Hardwood, Mineral Wool, Plaster dividers \& Glass fleece.

\section{System Design and Specification}

For conducting the experiment, rectangular acoustic tunnel with seven chambers has been designed and fabricated which is given in Figure 1.1. This consists of seven chambers with different material and specification to reduce the sound level. Specification of tunnel is $1219.2 * 177.8 * 177.8 \mathrm{~mm}$ Which is fitted with force draught fan at outlet to supply air which passes through hot chamber. This acoustic tunnel is divided into seven different chambers.

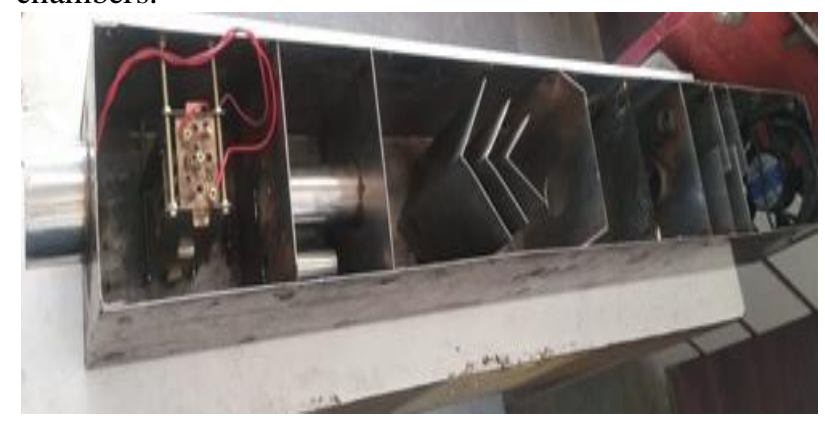

Figure 1.1 : Acoustic Model

1.1.1Hot Chamber-Chamber one contains a plates of mild steel of size $170 * 170 \mathrm{~mm}$ with a long pipe attached at center of $55 \mathrm{~mm}$ length and $76.2 \mathrm{~mm}$ diameter with another short pipe attached with center of $27.5 \mathrm{~mm}$ length and $38.1 \mathrm{~mm}$ diameter. Material used for the plate is mild steel with thickness of $1.63 \mathrm{~mm}$. 


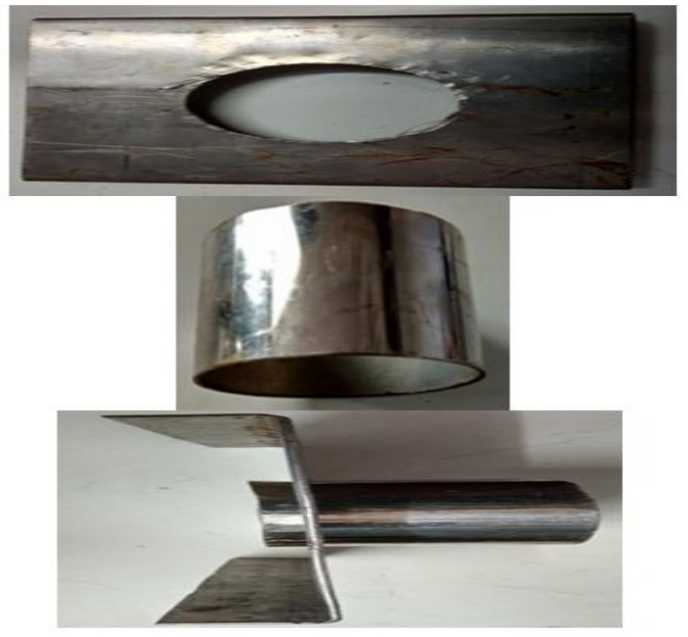

Figure 1.2 : Hot Chamber

1.1.2 Characteristics of Cylindrical Pipe ChamberSecond chamber consists of plates of mild steel of size $170 \mathrm{~mm} * 170 \mathrm{~mm}$. Both plates are separated at a distance of $110 \mathrm{~mm}$ through a pipe of $50.8 \mathrm{~mm}$ diameter at center. Corner pipe of length $70 \mathrm{~mm}$ and $25.4 \mathrm{~mm}$ diameter is attached at one of the plate.

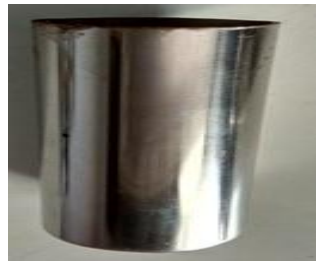

Figure 1.3: Cylindrical Pipe Chamber

1.1.3 Characteristics of Triangular Passage Chamber-To divert the flow of air and check the effect on sound level in $\mathrm{db}$. Third chamber is designed which consists three right angle passage fabricated with mild steel of sizes $170 * 180$ $\mathrm{mm}, 170 * 150 \mathrm{~mm}$, and $170 * 130 \mathrm{~mm}$ Material used for the plate is mild steel with thickness of $1.63 \mathrm{~mm}$.

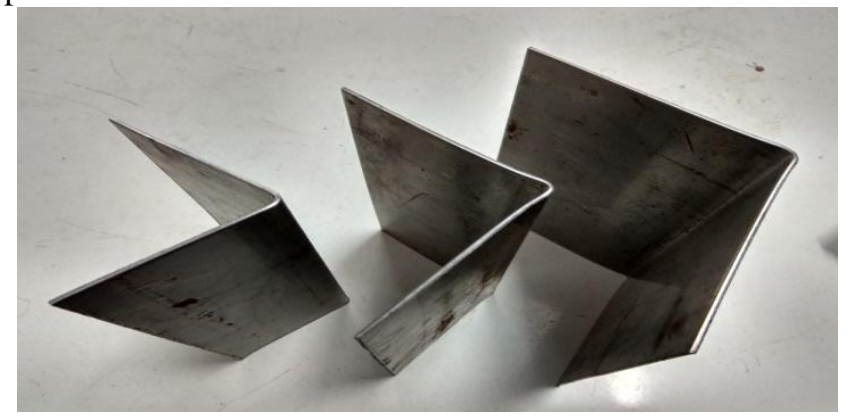

Figure 1.4: Triangular Passage Chamber

1.1.4 Characteristics of Tapered Plate Chamber-Fourth chamber contain a plate of mild steel with size of $170 * 230$ $\mathrm{mm}$. The plate is tapered at $47.5 \mathrm{~mm}$ from both sides at an angle of 60 degree approx and having diameter of $76.2 \mathrm{~mm}$. Thickness of the plate is $1.63 \mathrm{~mm}$.

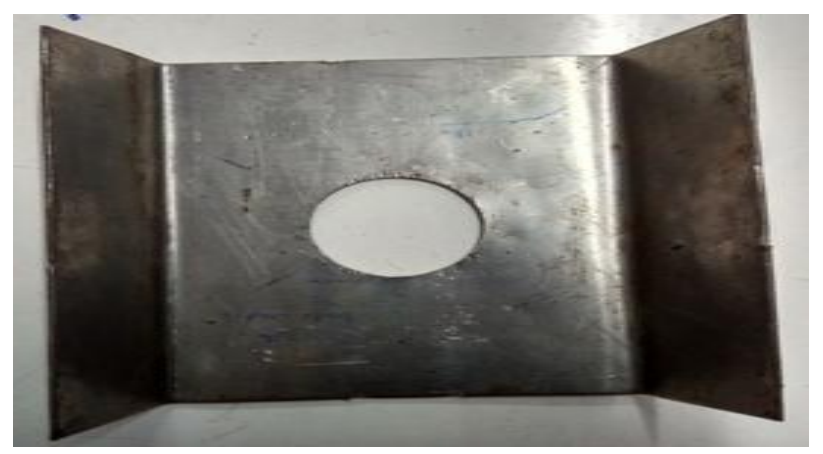

Figure 1.5: Tapered Plate Chamber

1.1.5 Characteristics of Net Chamber-Fifth chamber contains a net of $170 * 170 \mathrm{~mm}$. Material used is mild steel and hole size is $6 \mathrm{~mm}$ and thickness of $1.63 \mathrm{~mm}$.

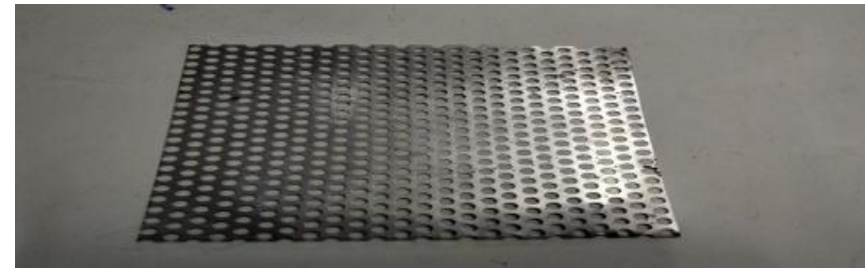

Figure 1.6 : Net Chamber

1.1.6 Characteristics of Diagonal Plate Chamber-Sixth chamber contains a plate of size $170 * 170 \mathrm{~mm}$ with 3 pipes arranged diagonally. The length of all three pipes is $30 \mathrm{~mm}$ and diameter of center pipe is $50.8 \mathrm{~mm}$ while diameter of both corner side pipes is $25.4 \mathrm{~mm}$. Thickness of the pipe is $1.02 \mathrm{~mm}$. Material used for plate is Mild Steel and for pipe is Stainless Steel 204.

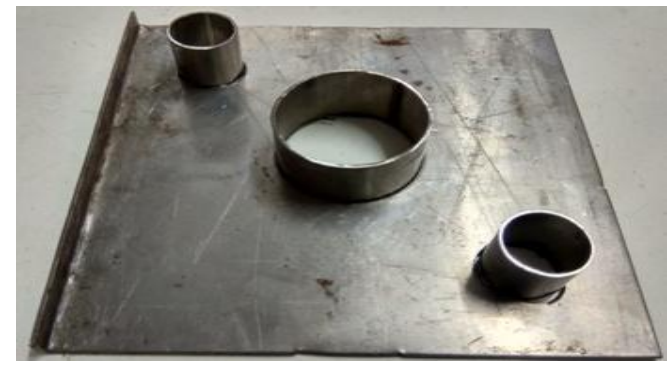

Figure1.7: Diagonal Plate Chamber

1.1.7 Characteristics of Zigzag Plate Chamber-Seventh chamber contains zigzag plates of size $170 * 100 \mathrm{~mm}$ each. Thickness of the plate is $1.63 \mathrm{~mm}$. Material used for plate is Mild Steel.

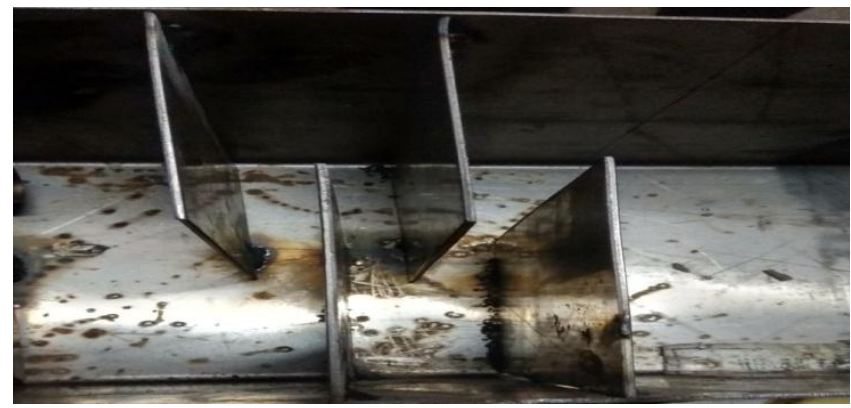

Figure 1.8: Zigzag Plate Chamber

1.1.8 Characteristics of Fan-In our design we have used a fan operating at 220V, $50 \mathrm{~Hz}$ AC and 0.29A. Size of the Fan is $170 * 170 * 50 \mathrm{~mm}$.

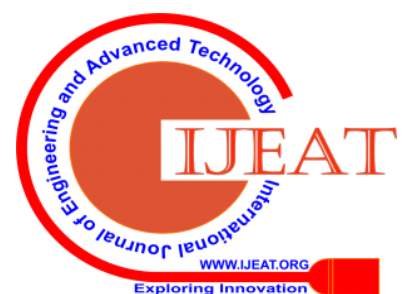




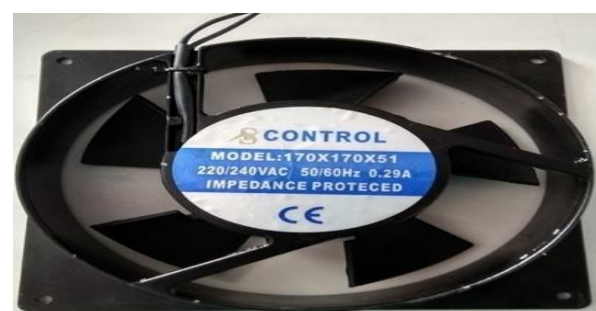

Figure 1.9: Silencer Fan

\section{RESULT}

Table 1 Transmission loss using noise barrier in $\mathrm{dB}$

\begin{tabular}{|l|l|l|}
\hline SPL (Source) $=96$ & SPL (Receiver) $=$ & $\begin{array}{l}\text { TL }=\text { SPL source }- \\
\text { SPL receiver (96- } \\
65=31)\end{array}$ \\
\hline
\end{tabular}

The complete noise level reading at source and receiver is shown in Table 1. After placing noise level meter at this respective place when the system is started it first takes the reading of Sound Pressure Level (SPL) at source $\&$ then at receiver. After taking reading the transmission loss is calculated.

\section{CONCLUSION}

We have studied muffler designing methods. After studying this methods and procedures for designing a muffler, we conclude that noise barrier chamber is more efficient than other types of mufflers. A sound absorbing chamber is an efficient device for achieving important attenuation. A chamber located at the fan discharge can be an effective and economical way to decrease noise entering the duct system.

\section{REFERENCES}

1. Z.Fang And C.Y Liu, "Combined mesh free method and mode matching approach for transmission loss predictions of expansion chamber silencers, Wuhan, Hubei, China", Elsevier : (Pg 168-177), 2017.

2. D.P. Jena and S.N. Panigrahi, "Numerically Estimating Acoustic Transmission Loss Of A Reactive Muffler With And Without Mean Flow, Rourkela, India", Elsevier : (Pg 168-186), 2017.

3. Ahmed Elsayed and Christophe Bastien (et.al), "Investigation Of Baffle Configuration Effect On The Performance Of Exhaust Mufflers, London, U.K”, Elsevier : (Pg 86-94), 2017.

4. Chenzhi Cai, Cheuk Ming Mark and Xu Wang, "Noise Attenuation Performance Improvement By Adding Helmholtz Resonators On The Periodic Ducted Helmholtz Resonator System, Hung Hom, Kowloon, Hong Kong, China", Elsevier : (Pg 8-15), 2017.

5. Shen Chao and Hou Liang, "Comparison Of Various Algorithms For Improving Acoustic Attenuation Performance And Flow Characteristic Of Reactive Mufflers, Xiamen, China", Elsevier : (Pg 291-296), 2017.

6. D.D Zhu and Z.L. Ji, "Transmission Loss Prediction Of Reactive Silencers Using 3-D Time-Domain CFD Approach And Plane Wave Decomposition Technique, Harbin, Heilongjiang, PR China", Elsevier : (Pg 25-31), 2016.

7. Zhenbo Lu, Yongdong. Cui and Marco Debiasi, "Active MembraneBased Silencer And Its Acoustic Characteristics, Singapore", Elsevier : (Pg 39-48), 2016.

8. Valdimir B. Tupov "Absorptive Silencers Of Foeced-Draft Fans With Improved Performance, Moscow, Russia”, ICSV22 : (Pg 1-8), 2015.

9. D.P. Jena and S.N. Panigrahi, "Estimating Acoustic Transmission Loss Of Perforated Filters Using Finite Element Method, Hyderabad, India", Elsevier : (Pg 1-14), 2015.

10. S.N Panigrahi and M.L Munjal, "A Generalized Scheme For Analysis Of Multifarious Commercially Used Mufflers, Bangalore, Karnataka, India", Elsevier : (Pg 660-681), 2007.

\section{AUTHORS PROFILE}

Dr. Sandeep Yadav (Ph.D). (Mechanical Engineering), Assistant Professor, Department of Fire $\begin{array}{lll}\text { Technology \& } & \text { Safety }\end{array}$ Engineering Medi-Caps University, Indore, India

Mr. Rituraj Raghuvanshi (M.Tech) (Industrial Safety Engineering), Assistant Professor, Department of Fire Technology \& Safety Engineering Medi-Caps University, Indore, India 